\title{
Paroxysmal tachycardia in a patient after the Senning procedure for transposition of the great arteries
}

\author{
Marek Jastrzębski ${ }^{1, A-B, D}$, Danuta Czarnecka ${ }^{2, E}$ \\ A - Research concept and design, B - Collection and/or assembly of data, C - Data analysis and interpretation, \\ D - Writing the article, E - Critical revision of the article, F - Final approval of article
}

1 First Department of Cardiology, Interventional Electrocardiology and Hypertension, Jagiellonian University Medical College, Cracow, Poland. 2 First Department of Cardiology, Interventional Electrocardiology and Hypertension, Jagiellonian University Medical College, Cracow, Poland.

Address for correspondence:

Marek Jastrzębski

email: mcjastrz@cyf-kr.edu.pl

First Department of Cardiology, Interventional Electrocardiology and Hypertension, Jagiellonian University Medical College, Cracow, Poland.

Danuta Czarnecka

email: dczarnecka@interia.pl

First Department of Cardiology, Interventional Electrocardiology and Hypertension, Jagiellonian University Medical College, Cracow, Poland.

\begin{abstract}
We describe a 19-year- old man with transposition of the great arteries, which had been corrected 14 years earlier with the Senning procedure, who experienced recurrent atrioventricular nodal reentrant tachycardia (AVNRT). The ablation
\end{abstract}

procedure is described with a discussion and literature review concerning the possible and preferred slow pathway ablation strategy in such patients.

\section{Key words:}

\section{Case presentation}

We describe a 19-year- old man with transposition of the great arteries, which had been corrected 14 years earlier (Senning procedure), who experienced recurrent tachycardia with incomplete right bundle branch block QRS morphology. Since the QRS morphology was identical to that during sinus rhythm and typical of patients with transposition of the great arteries (prominent features of right ventricular hypertrophy), he was presumptively diagnosed with supraventricular tachycardia (Figure 1). Complex cardiosurgical operations involving atrial tissue predispose to the occurrence of intraatrial reentry due to iatrogenic scars. ${ }^{[1]}$ In the current case, however, the electrophysiological study resulted in a diagnosis of atrioventricular reentrant tachycardia (AVNRT) - Figure 2, panel A. The tachycardia had a cycle length of 380-520 ms, and a ventriculo-atrial interval of $60 \mathrm{~ms}$ was observed. Moreover, there was a $\mathrm{V}-\mathrm{A}-\mathrm{V}$ response to ventricular overdrive pacing, and the post-pacing interval was $158 \mathrm{~ms}$ longer than the tachycardia cycle length. With the support of a 3D system (EnSite NavX St. Jude Medical, Inc., St. Paul, MN, USA) we first tried to ablate the AVNRT substrate in the venous atrium near the His bundle potential with access via the inferior vena cava (Figure 2, panel B and Figure 3, panel A), but this attempt was unsuccessful and hazardous (non-conducted nodal ectopic beats during ablation with transient $\mathrm{AH}$ interval prolongation). It seemed that the venous atrium allowed access only to the upper part of the triangle of Koch with the His bundle and the fast atrioventricular node pathway. Therefore, a retrograde aortic approach to the systemic ventricle and then via the tricuspid valve to the area of typical localization of the slow pathway in the remnant of the anatomic right atrium (Figure 2, panel C and Figure 3, panel B, black arrow) was used. An ablation catheter was positioned on the His bundle potential and pushed deeper into the atrium in a direction inferior and lateral to the His bundle potential to search for a small fragmented atrial potential. Application of radiofrequency current energy in this position (Figure 2, panel C, red dots) resulted in an accelerated nodal rhythm and arrhythmia non-inducibility, with no recurrences during 2 years of follow-up. Figure 2, panel $\mathrm{D}$ illustrates the anatomical route that can be used to 
access the slow pathway region with the ablation catheter in a patient with such an unusual anatomy. Procedure duration,
RF time, fluoroscopy time and X-ray dose were $95 \mathrm{~min}, 8 \mathrm{~min}$, $22 \mathrm{~min}$ and $134 \mathrm{mGy}$, respectively.

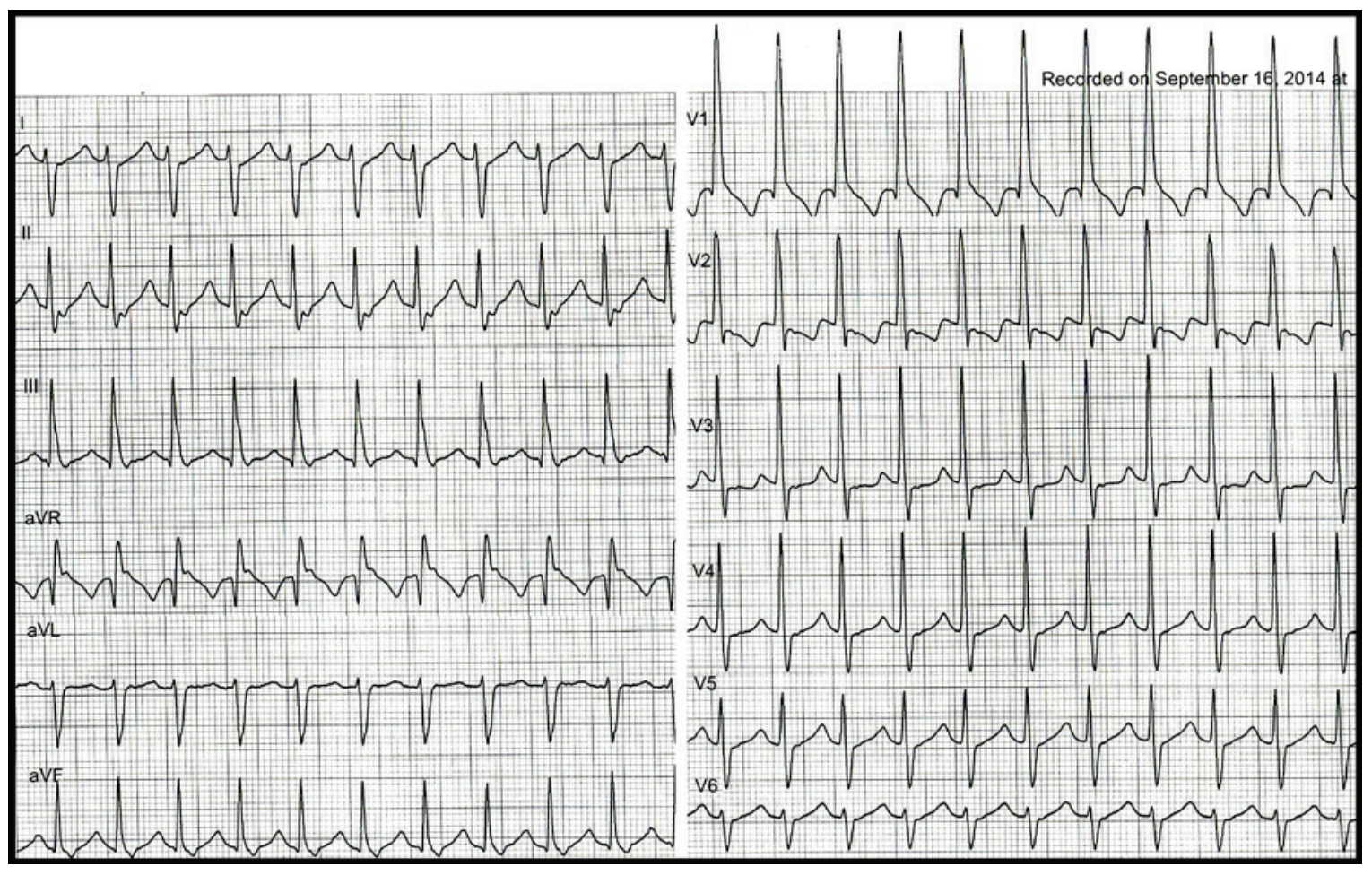

Figure 1. 12-lead ECG with clinical tachycardia: a regular, narrow QRS complex tachycardia of 150 bpm with probable ' $p$ ' waves visible immediately after QRS complexes in leads II, III, aVF. Prominent R wave present in leads V1-V2 indicates right ventricular hypertrophy. Paper speed $25 \mathrm{~mm} / \mathrm{s}$.

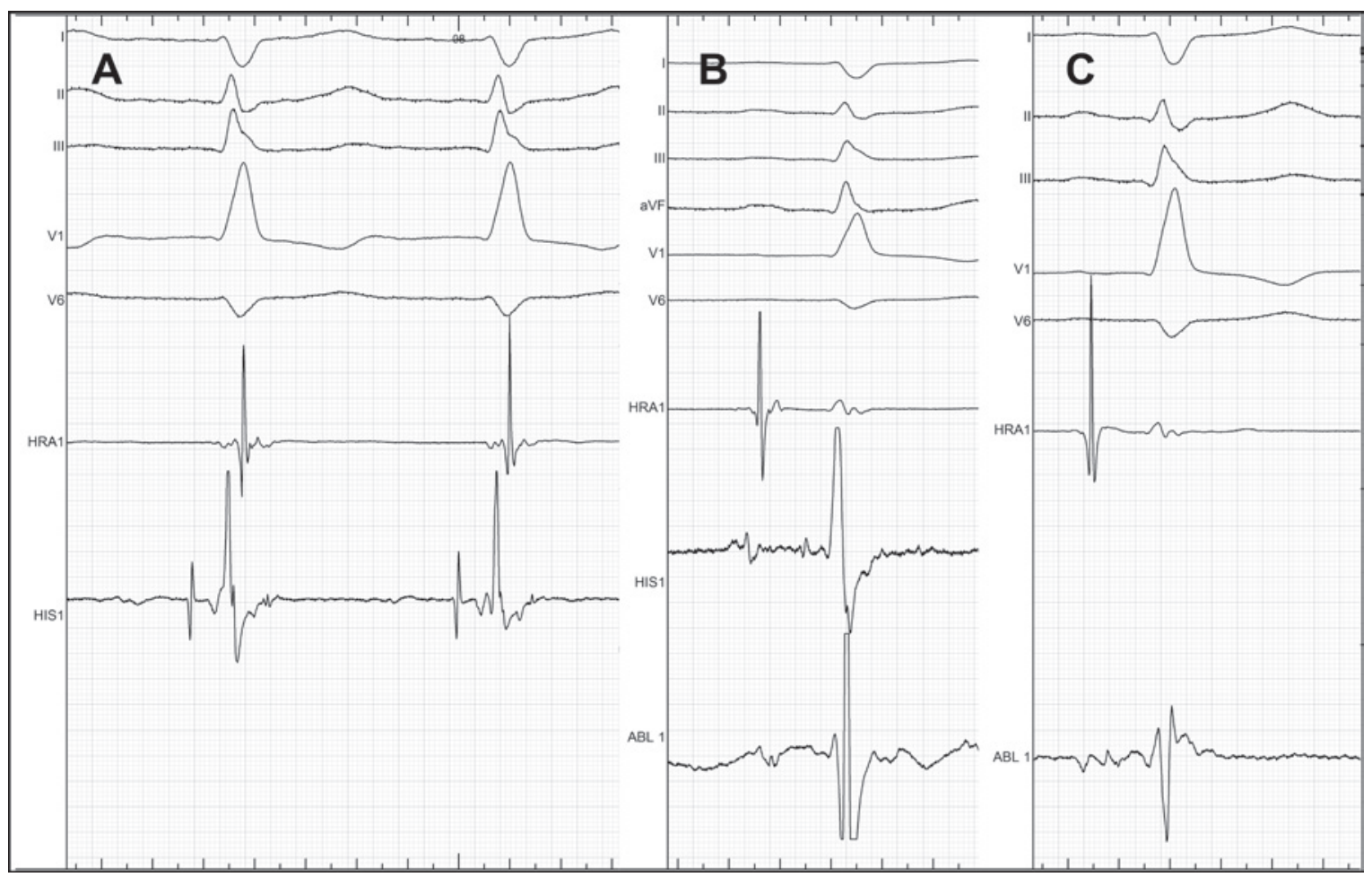

Figure 2. Surface ECG leads I, II, III, V1, V6 and intracardiac tracings: panel A - induced atrioventricular nodal reentrant tachycardia, panel B - site of the unsuccessful ablation attempt on the venous site of the triangle of Koch, panel C - site of the successful ablation attempt on the arterial site of the triangle of Koch. ABL1 - ablation catheter, HIS1 - His bundle catheter, HRA1 - right atrial catheter. Paper speed $100 \mathrm{~mm} / \mathrm{s}$. 


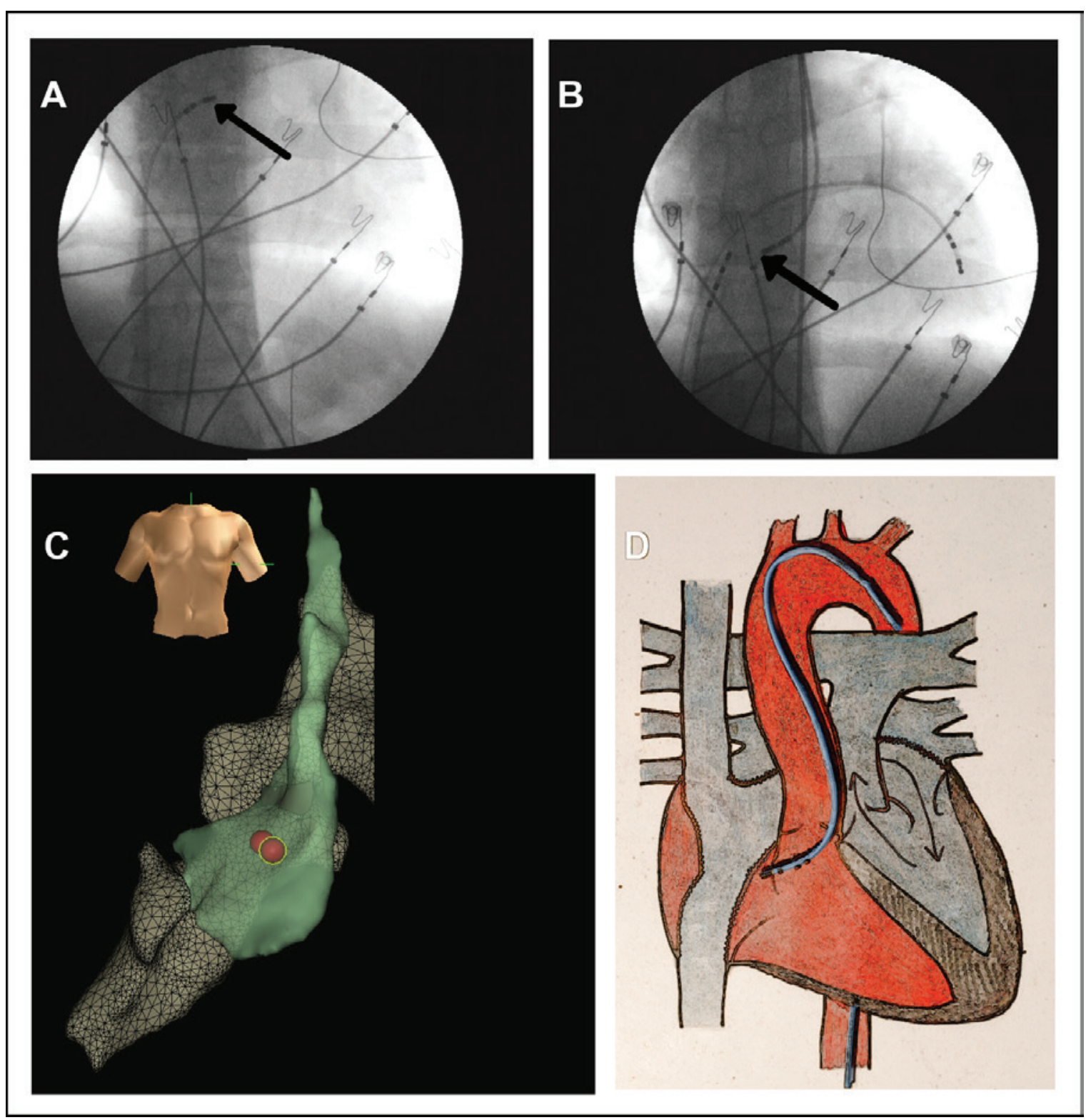

Figure 3. Panel A: fluoroscopic projection in the postero-anterior view illustrating the ablation catheter in the systemic venous atrium at the unsuccessful ablation site (black arrow). Panel B: right anterior oblique view showing the ablation catheter (black arrow) positioned via the aorta in the slow pathway region at the successful ablation site. Panel C: 3D system (EnSite NavX St. Jude Medical, Inc., St. Paul, MN, USA) partial reconstruction of the venous atrium (dotted surface) and arterial approach (green surface) with red dots at the successful ablation site. Panel D: schematic illustration of the anatomy and the ablation catheter route.

\section{Commentary}

Supraventricular cardiac arrhythmias significantly contribute to morbidity and mortality in patients after Senning or Mustard operations. ${ }^{[2]}$ The most commonly diagnosed arrhythmia is intraatrial reentrant tachycardia (atypical atrial flutter or micro/macro reentrant atrial tachycardia) occurring in approximately $30 \%$ of patients in long-term follow-up. ${ }^{[23]}$ However, AVNRT should also be high on the list of possible diagnoses, as in the published short series of patients after Senning or Mustard operations who have undergone electrophysiological study for supraventricular tachycardia AVNRT was identified in 3 of 11 patients $(27 \%)$ in the series of Kanter et al., ${ }^{[4]} 4$ of $34(12 \%)$ in the study of Wu et al., ${ }^{[5]}$ while Greene et al. reported AVNRT in 4 of 18 patients $(22 \%){ }^{[6]}$ - with the remaining patients from these series diagnosed with intraatrial reentry. Despite being supraventricular, these arrhythmias may cause acute cardiac failure or sudden cardiac death due to the fast ventricular response. ${ }^{[2]}$

There are very limited data on AVNRT ablation in patients with transposition of the great arteries who have undergone the Senning operation, making the optimal approach for this procedure difficult to determine. Access to the typical anatomical location of the slow pathway via the classical route (i.e. via the inferior vena cava to the right atrium) is usually impossible because, in the Senning operation, this part of the right atrium is surgically sequestered from the anatomical/venous right 
atrium and used to redirect blood from the pulmonary veins to the systemic ventricle (anatomic right ventricle). Therefore ablation of AVNRT in a patient who has undergone the Senning/Mustard operation requires non-standard approaches. Three access options can be considered in order to modify atrioventricular node physiology: $1 /$ access to the systemic venous atrium; 2 / the transbaffle approach to the triangle of Koch, and 3/ the retrograde aortic approach. In the experience of Greene et al., access via the femoral vein to the systemic venous atrium despite induction of junctional tachycardia during ablation is not successful in eliminating AVNRT; we also failed using this approach. Venous access can be successful when it is combined with a transbaffle access, although this requires either a baffle leak or a rather invasive strategy with a baffle puncture. ${ }^{[7,8]}$ In contrast, retrograde aortic access is relatively easy and, judging by the current case and the published series (albeit mainly in patients after the Mustard procedure), often results in a curative ablation.

In conclusion, the transaortic retrograde approach to the remnant of the anatomically right atrium should be the initially preferred slow pathway ablation strategy in patients after the Senning procedure.

\section{References}

1. Gelatt M, Hamilton RM, McCrindle BW, et al. Arrhythmia and mortality after the Mustard procedure: a 30-year single-center experience. J Am Coll Cardiol 1997 Jan;29(1):194-201.

2. Kammeraad JA, van Deurzen $\mathrm{CH}$, Sreeram N, et al. Predictors of sudden cardiac death after Mustard or Senning repair for transposition of the great arteries. J Am Coll Cardiol 2004 Sep 1;44(5):1095-102.
3. Warnes CA, Somerville J. Transposition of the great arteries: late results in adolescents and adults after the Mustard procedure. Br Heart J 1987 Aug;58(2):148-55.

4. Kanter RJ, Papagiannis J, Carboni MP, Ungerleifer RM, Sanders WE, Wharton JM. Radiofrequency catheter ablation of supraventricular tachycardia substrates after Mustard and Senning operations for d-transposition of the great arteries. J Am Coll Cardiol 2000 Feb;35(2):428-41.

5. Wu J, Deisenhofer I, Ammar S, et al. Acute and longterm outcome after catheter ablation of supraventricular tachycardia in patients after the Mustard or Senning operation for D-transposition of the great arteries. Europace 2013 Jun;15(6):886-91.

6. Greene AE, Skinner JR, Dubin AM, Collins KK, Van Hare GF. The electrophysiology of atrioventricular nodal reentry tachycardia following the Mustard or Senning procedure and its radiofrequency ablation. Cardiol Young 2005 Dec;15(6):611-6.

7. McCanta AC, Kay JD, Collins KK. Cryoablation of the slow atrioventricular nodal pathway via a transbaffle approach in a patient with the Mustard procedure for d-transposition of the great arteries. Congenit Heart Dis 2011 Sep;6(5):479-83.

8. Jones DG, Jarman JWE, Lyne JC et al. The safety and efficacy of trans-baffle puncture to enable catheter ablation of atrial tachycardias following the Mustard procedure: A single centre experience and literature review. Int J Cardiol. 2013 Sep 30; 168(2):1115-20. 